\title{
PERCEPÇÃO DOS ESTUDANTES DE MEDICINA VETERINÁRIA SOBRE A FORMAÇÃO E ATUAÇÃO EM SAÚDE PÚBLICA NO ÂMBITO DA UNIVERSIDADE FEDERAL DE PELOTAS-RS
}

TELES, Alessandra Jacomelli ${ }^{1}$;

LIMA, Jackeline Vieira ${ }^{2}$; VEECK, Natiele Isaura de Almeida ${ }^{2}$; ALMEIDA, Rodrigo Bozembecher de ${ }^{2}$; GUIMARÃES, Tássia Gomes ${ }^{3}$; GIROLOMETTO, Giovani ${ }^{4}$;

\footnotetext{
${ }^{1}$ MV, Especialista em Saúde Coletiva, MSc., Doutoranda do Programa de Pós-Graduação em Veterinária/UFPEL;

${ }^{2}$ Graduando em Medicina Veterinária, Faculdade de Veterinária/UFPEL; ${ }^{3} M V$, Especialista em Saúde Coletiva, MSc. em Ciências Veterinárias/UFPEL; ${ }^{4}$ MV, Dr. em Ciências veterinárias, Faculdade de Veterinária/UFPEL; ${ }^{5} M V$, MSc., Dr. Professor do Departamento de Medicina Veterinária Preventiva, Faculdade de Veterinária/UFPEL.
}

\section{RESUMO}

A responsabilidade da medicina veterinária em contribuir para promoção do bem-estar da sociedade é cada vez maior, portanto, a preocupação com a formação acadêmica na área de saúde pública se faz necessária. O presente estudo objetivou fazer uma análise sobre o ensino em saúde pública, através da percepção de estudantes de medicina veterinária sobre a atuação do médico veterinário na área. Para a identificação das noções dos estudantes foi aplicado um questionário individual, para os acadêmicos. A pesquisa demonstrou que o perfil dos estudantes é composto na sua maioria por mulheres e a admiração pela carreira é o principal motivo para escolha do curso. Os acadêmicos reconheceram a relevância da profissão na área de saúde pública, mas desconhecem como se dá essa atuação e que este tema esteja inserido na grade curricular do curso de graduação. Não foi observado evolução do aprendizado ao longo do curso, demonstrando a necessidade da reestruturação curricular do curso. É necessário que os graduandos adquiram o conhecimento durante a etapa de formação para que a profissão ocupe o espaço devido no âmbito da área de saúde, com estratégias pedagógicas que incluam o estudante no serviço de saúde.

Palavras-chave: Ensino. Graduação. Educação em saúde. Saúde coletiva. 


\section{INTRODUÇÃO}

A medicina veterinária com os seus saberes tem como objetivo final da profissão o bemestar das populações humanas por meio do cuidado com a saúde animal, as habilidades e recursos veterinários são aplicados em benefício da saúde do homem, o qual faz parte de um ecossistema e mantém constante relação com os animais (PFUETZENREITER; ZYLBERSZTAJN, 2008). É neste ambiente em que o homem realiza todas as atividades para sua sobrevivência, produz, transforma e consome alimentos, bens e serviços, cria animais, e onde está exposto a todo tipo de riscos à saúde decorrente, por diversas vezes, da sua ação ou da relação que tem com o meio em que vive. Tudo isto faz com que o papel do médico veterinário seja importante na sociedade e na ciência (RAMOS, 2008).

A saúde pública reúne as práticas e conhecimentos organizados para execução de ações e medidas que evitem, reduzam e/ou minimizem agravos à saúde, assegurando condições para a manutenção e sustentação da vida (SABROZA, 1994). A medicina veterinária é uma profissão da área da saúde, a partir de 2011 isto foi fundamentado com a incorporação do médico veterinário nas equipes profissionais dos Núcleos de Apoio à Saúde da Família (NASF), institucionalizadas pelo Ministério da Saúde e geridas pelo Sistema Único de Saúde (SUS) (BRASIL, 2011).

A formação em medicina veterinária caracteriza-se por uma orientação pela doença e reabilitação, em que os alunos aprendem a ver com o olhar do estilo de pensamento associado à medicina curativa e individual (PFUETZENREITER; ZYLBERSZTAJN, 2008). Ainda que o ensino deva garantir a formação de um profissional com perfil generalista, humanista, apto a compreender e traduzir as necessidades de indivíduos e comunidades (BRASIL, 2003).

O curso de medicina veterinária da Universidade Federal de Pelotas (UFPEL) tem a duração mínima de cinco anos, está estruturado em 10 semestres letivos. A carga horária total do curso é de 4930 horas, sendo 4250 horas distribuídas nas 55 disciplinas obrigatórias e 680 horas de estágio curricular.

Mesmo com significativa demanda por profissionais veterinários especializados em Saúde Pública, alguns estudos demonstram que as escolas de medicina veterinária não têm enfatizado a capacitação nessa área (BÜRGER, 2010; PFUETZENREITER, 2003). A graduação é 
o primeiro contato que o futuro profissional tem com a área, para tanto é imprescindível que as atividades do médico veterinário em Saúde Pública sejam cada vez mais enfatizadas durante a formação. Por esse motivo, a fim de ocupar o lugar conquistado na área da saúde há a necessidade de que tal formação garanta o nível de competência consistente com as demandas da sociedade, visto que o reconhecimento da profissão está na dependência de sua relevância social.

Diante do exposto, este trabalho objetivou identificar as noções de acadêmicos de medicina veterinária sobre a atuação do médico veterinário em Saúde Pública.

\section{MATERIAL E MÉTODOS}

No presente estudo foi realizado um levantamento de caráter quantitativo, descritivo de acordo com Marconi e Lakatos (2010).

\section{Sujeitos}

Os sujeitos da pesquisa foram os estudantes de medicina veterinária da Universidade Federal de Pelotas. Por semestre ingressam no curso 66 acadêmicos e atualmente são 664 matriculados. A amostra foi obtida dos estudantes em três etapas diferentes do curso:

1 - no início da graduação, primeiro ano, (primeiro e segundo semestres);

2 - no terceiro ano (quinto e sexto semestres), quando tem início as disciplinas profissionalizantes;

3 - no quinto ano (nono semestre), próximo ao término do curso e anterior ao estágio curricular obrigatório.

Os estudantes foram abordados durante as disciplinas listadas na Tabela 1 . Todos os graduandos presentes responderam o questionário, buscou-se realizar a pesquisa em um período que não correspondesse com provas e para que não atrapalhasse o andamento da disciplina e também buscando atingir um número maior de participantes, os questionários foram aplicados no início da aula ou anterior ao intervalo, quando presente. 
Tabela 1 - Disciplinas em que os estudantes de medicina veterinária da Universidade Federal de Pelotas foram entrevistados.

\begin{tabular}{llll}
\hline \multirow{2}{*}{ Grupo } & $\begin{array}{l}\text { Semestre } \\
\text { letivo }\end{array}$ & Disciplinas & $\begin{array}{l}\text { Estudantes } \\
\text { (n) }\end{array}$ \\
\hline \multirow{2}{*}{1} & 19 & Iniciação à Medicina Veterinária & 57 \\
& $2 \underline{0}$ & Anatomia dos Animais Domésticos II & 46 \\
2 & 50 & Epidemiologia e Ecologia & 42 \\
3 & 60 & Doenças Infecciosas & 57 \\
& 9 o & Zoonoses, Administração Sanitária e Saúde Pública & 27 \\
\hline
\end{tabular}

Anterior a aplicação do questionário, os entrevistados foram informados da realização da pesquisa genericamente, mas os detalhes foram explicados após responderem, dando a liberdade para que ainda neste momento pudessem solicitar a sua exclusão.

\section{Coleta de dados}

A coleta de dados foi realizada por meio de questionários individuais semi-estruturados. 0 questionário era composto por três partes: I - perfil do estudante de graduação em medicina veterinária; II - ensino em saúde pública veterinária e III - conhecimento sobre atividades desenvolvidas pelo médico veterinário em saúde pública veterinária.

\section{Análise dos dados}

A análise dos resutados foi realizada através da distribuição da frequências das respostas dadas pelos acadêmicos e teste de Qui-quadrado, através do programa disponibilizado online Social Science Statistics (2017), onde p-valores inferiores a 0,05 foram considerados estatisticamente significativos.

\section{Aspectos éticos}

O questionário aplicado foi submetido à análise do Comitê de Ética em Pesquisa com Seres Humanos da Universidade Federal de Pelotas, sendo aprovado para sua aplicação (CAEE: 55247916.0.0000.5317). Os participantes concordaram com a pesquisa assinando o termo de consentimento livre e esclarecido, conforme exige o comitê de ética referido. 


\section{RESULTADOS E DISCUSSÃO}

Foram entrevistados 229 graduandos do curso de medicina veterinária da UFPEL (45\% do total de alunos do curso), 103 do primeiro ano, 99 do terceiro ano e 27 do quinto ano.

As primeiras perguntas do questionário objetivaram traçar o perfil dos estudantes. Observou-se que do total dos entrevistados, $77,7 \%$ eram do sexo feminino, enquanto que $22,3 \%$ eram do sexo masculino, demonstrando que as mulheres estão conquistando um grande espaço no mercado de trabalho, optando por profissões antes tidas apenas como masculinas. A diversificação das atividades ocorridas na profissão a partir dos anos 1990 resultou em forte estímulo para novos ingressantes nos cursos de medicina veterinária, sobretudo os do sexo feminino (MONDADORI et al., 2013). O perfil dos estudantes de medicina veterinária sofreu alterações ao longo dos anos, grande parte são mulheres provenientes do meio urbano (BÜRGER, 2010). Igualmente, o predomínio feminino é observado nos programas de residência, bem como o número de novas inscrições no Conselho de Medicina Veterinária é composto principalmente por mulheres, atingindo os 60\% (CFMV, 2015).

A maioria dos graduandos entrevistados em nosso estudo são oriundos do estado do Rio Grande do Sul (82,5\%), desses, $37 \%$ são do município de Pelotas, onde se localiza a Universidade. A adesão da UFPEL ao Sistema de Seleção Unificada (SISU) também pode contribuir para mudança no perfil dos acadêmicos. Uma avaliação da Universidade Federal de Minas Gerais (UFMG) confirma o crescimento do número de estudantes de outros estados e do interior, após ingresso pelo SISU (EEFFTO, 2015).

Os estudantes foram questionados sobre os motivos que os levaram a optar pela medicina veterinária. A maioria dos entrevistados tendeu para lado afetivo e de preferência pessoal, "admiração pela carreira" 36,7\% foi a alternativa mais assinalada, seguida de "gostar de animais" 35,8\%, além da "convivência com o meio rural" 17,7\%. Aspectos financeiros e familiares são pouco levados em consideração quando comparados aos anteriores, demonstrando que o futuro profissional desenvolverá suas atividades com prazer e dedicação (Tabela 2). 
Tabela 2 - Motivo da escolha do curso de graduação dos estudantes de medicina veterinária da Universidade Federal de Pelotas.

\begin{tabular}{lll}
\hline Motivo & $\mathrm{n}^{*}$ & $\%$ \\
\hline Admiração pela carreira & 79 & $36,7 \%$ \\
Gosto por animais & 77 & $35,8 \%$ \\
Convivência com o meio rural & 38 & $17,7 \%$ \\
Mercado favorável & 8 & $3,7 \%$ \\
Influência familiar & 6 & $2,8 \%$ \\
Outro motivo & 7 & $3,3 \%$ \\
\hline TOTAL & 215 & $100 \%$
\end{tabular}

* Estudantes que responderam mais de uma alternativa tiveram a questão anulada $(n=14)$.

A motivação pela escolha profissional também foi avaliada por outros autores. Resultados muito semelhantes foram encontrados por Bürger (2010), sendo a principal escolha dos graduandos em seu estudo, a admiração pela carreira (42,3\%), seguida da afinidade com espécies animais (31,9\%) e convivência com o meio rural $(13,6 \%)$. Em contrapartida Pfuetzenreiter (2003) obteve resultados diferentes do presente estudo, relatando o principal motivo ser o contato com o meio rural ou com atividades relacionadas à pecuária (50\%), influência familiar (40\%) e gosto pelos animais (20\%). Pode-se observar que em quase uma década a motivação dos ingressantes mudou, evidenciando a modificação do perfil dos acadêmicos de medicina veterinária ao longo dos anos. Até a metade do século XX, a maioria dos estudantes possuía forte vínculo com o meio rural, a partir da década de 80 a medicina veterinária sofreu um intenso processo de urbanização, passando a enfatizar áreas como a clínica e a cirurgia de animais de companhia (MONDADORI et al., 2013).

Na sequência o questionário buscou caracterizar o ensino de saúde pública veterinária. $\mathrm{Na}$ opinião dos estudantes entrevistados o profissional médico veterinário está habilitado a trabalhar na área da saúde pública veterinária, sendo que 95,2\% se mostraram cientes dessa ser uma alternativa de trabalho para o médico veterinário, e $98,7 \%$ relataram ser relevante a atuação nessa área. 
Embora a maioria dos entrevistados afirme que o médico veterinário possui formação para atuar em saúde pública, a metade $(49,6 \%)$ desconhece que o tema esteja inserido na matriz curricular do curso de graduação (Tabela 3). O curso de medicina veterinária da UFPEL possui sete disciplinas diretamente ligadas à saúde pública veterinária (Tabela 4), desta forma é evidente que os estudantes não têm conhecimento da referida área, significado e atividades a serem desenvolvidas.

Tabela 3 - Reconhecimento dos estudantes sobre a inserção do tema saúde pública veterinária na matriz curricular do curso de Medicina Veterinária da Universidade Federal de Pelotas.

\begin{tabular}{lllll}
\hline Resposta & 10 ano & 3\% ano & 5o ano & Total \\
& $\mathrm{n}(\%)$ & $\mathrm{n}(\%)$ & $\mathrm{n}(\%)$ & $\mathrm{n}(\%)$ \\
\hline Sim & $18(17,6)$ & $38(39,2)$ & $18(66,7)$ & $74(32,7)$ \\
Não & $14(13,7)$ & $17(17,5)$ & $9(33,3)$ & $40(17,7)$ \\
Não sabe & $70(68,7)$ & $42(43,3)$ & 0 & $112(49,6)$ \\
\hline Total & 102 & 97 & 27 & 226 \\
\hline
\end{tabular}

* Estudantes que não responderam a questão $(n=3)$ não foram contabilizados.

Tabela 4 - Disciplinas relacionadas com a área de saúde pública veterinária do curso de Medicina Veterinária da Universidade Federal de Pelotas.

\begin{tabular}{|c|c|c|}
\hline Disciplina & Semestre letivo & Carga horária (h) \\
\hline Tecnologia dos Produtos de Origem Animal & 40 & 85 \\
\hline Saneamento & 40 & 51 \\
\hline Epidemiologia e Ecologia & 50 & 68 \\
\hline Inspeção de Leite e Derivados & 9은 & 68 \\
\hline Inspeção de Carnes e Derivados & 9은 & 102 \\
\hline Inspeção de Pescados e Derivados & 9o & 68 \\
\hline Zoonoses, Administração Sanitária e Saúde Pública & 9은 & 68 \\
\hline
\end{tabular}

Ainda que quase a unanimidade dos graduandos afirme a importância do profissional atuar na área de saúde pública, tornou-se evidente o desconhecimento desses acerca das atividades desenvolvidas pela saúde pública veterinária e a real importância para a sociedade. Quando questionados sobre o conceito de saúde pública veterinária, apenas $28,51 \%$ optaram pela alternativa correta. Tal fato foi comprovado com a desinformação dos 
alunos quanto ao desenvolvimento de atividades desse profissional no SUS: $48,9 \%$ não tinham conhecimento do assunto, 13,1\% negaram e apenas $38 \%$ afirmaram tal participação (Tabela 5). Bürger (2010) descreve situações parecidas em seu estudo, em que os estudantes associam a área da saúde pública com controle de zoonoses, mas desconhecem a lei que inclui o médico veterinário na área da saúde. Mesmo que a participação do médico veterinário no NASF seja um fato atual, é importante que os futuros profissionais estejam cientes das possibilidades dentro da profissão (BRASIL, 2011).

Tabela 5 - Entendimento dos estudantes de graduação em medicina veterinária da Universidade Federal de Pelotas sobre a participação do médico veterinário no Sistema Único de Saúde.

\begin{tabular}{lllll}
\hline Resposta & $\begin{array}{l}\text { 10 ano } \\
\mathrm{n}(\%)\end{array}$ & $\begin{array}{l}\text { 30 ano } \\
\mathrm{n}(\%)\end{array}$ & $\begin{array}{l}\text { 50 ano } \\
\mathrm{n}(\%)\end{array}$ & $\begin{array}{l}\text { Total } \\
\mathrm{n}(\%)\end{array}$ \\
\hline Sim & $21(20,4)$ & $50(50,5)$ & $16(59,3)$ & $87(38,0)$ \\
Não & $20(19,4)$ & $9(9,1)$ & $1(3,7)$ & $30(13,1)$ \\
Não sabe & $62(60,2)$ & $40(40,4)$ & $10(37,0)$ & $112(48,9)$ \\
\hline Total & 103 & 99 & 27 & 229 \\
\hline
\end{tabular}

Na parte seguinte do questionário foram expostas situações com exemplos das atividades mais conhecidas popularmente do médico veterinário em saúde pública, com o objetivo de averiguar o conhecimento sobre a área estudada. Observou-se que a porcentagem de acertos das questões sobre a atuação do médico veterinário foi de $54,6 \%$ para vigilância em saúde; $57 \%$ para área de vigilância epidemiológica; 62,8\% para vigilância sanitária e 68,6\% para gestão e planejamento em saúde.

A avaliação da evolução do aprendizado, durante o desenvolvimento do curso, sobre os conceitos e atividades desenvolvidas pelo médico veterinário na área da saúde pública veterinária estão expostos na Tabela 6. Em relação ao tema vigilância em saúde, os alunos demonstraram uma evolução no aprendizado com o passar dos semestres letivos $(p<0,05)$. As áreas de vigilância sanitária e planejamento e gestão em saúde não demostraram diferenças na aquisição do conhecimento ao longo do curso. Contrariamente ao esperado, na área de vigilância epidemiológica os alunos da metade do curso demonstraram maior conhecimento que os alunos do último ano. 
Tabela 6 - Porcentagem de respostas corretas expressas pelos estudantes de medicina veterinária da Universidade Federal de Pelotas sobre as atividades do médico veterinário em saúde pública, de acordo com os diferentes anos do curso.

\begin{tabular}{lllll}
\hline \multirow{2}{*}{ Ano do curso } & $\begin{array}{l}\text { Vigilância em } \\
\text { saúde (\%) }\end{array}$ & $\begin{array}{l}\text { Vigilância } \\
\text { Epidemiológica (\%) }\end{array}$ & $\begin{array}{l}\text { Vigilância } \\
\text { Sanitária (\%) }\end{array}$ & $\begin{array}{l}\text { Planejamento } \\
\text { gestão em saúde (\%) }\end{array}$ \\
\hline 1 o & $47,1^{\mathrm{a}}$ & $42,6^{\mathrm{a}}$ & $60,2^{\mathrm{a}}$ & $68,6^{\mathrm{a}}$ \\
3ㅇ & $57,7^{\mathrm{a}}$ & $74,4^{\mathrm{b}}$ & $63,5^{\mathrm{a}}$ & $65,9^{\mathrm{a}}$ \\
5ㅇ & $74,1^{\mathrm{b}}$ & $55,6^{\mathrm{a}}$ & $70,4^{\mathrm{a}}$ & $77,7^{\mathrm{a}}$ \\
\hline
\end{tabular}

* Letras distintas na coluna indicam diferença estatística.

A análise de Bürger (2010) difere do presente estudo, em que os estudantes pesquisados adquiriram mais conhecimento sobre a área durante o curso de graduação. $O$ autor atribui essa evolução ao contato com diversas disciplinas e informações adquiridas com o passar dos semestres letivos e afirma que no último ano o aluno deve ter pleno conhecimento das atividades e da importância do profissional na área.

Quando avaliada a carga horária destinada a cada área de formação profissional dentro do currículo, conforme a classificação utilizada por Pfuetzenreiter e Zylberstajn (2004) e Bürger (2010), observou-se na matriz curricular do curso de medicina veterinária da UFPEL que 62,8\% são destinadas a clínica veterinária, 20,3\% a saúde pública veterinária e 16,9\% para zootecnia e produção animal. A maioria das disciplinas relacionadas com saúde pública veterinária (4 disciplinas) são ofertadas no penúltimo semestre. Evidencia-se uma discordância entre os resultados obtidos nesta pesquisa aliados a análise da matriz curricular. Sugerindo que os alunos do primeiro ano podem ter conhecimento prévio da área ou conhecimento adquirido das áreas de atuação profissional na disciplina "Introdução à Medicina Veterinária" (primeiro semestre). Presume-se que os acadêmicos do terceiro ano adquirem mais conhecimento com o desenvolvimento do curso, quando as outras três disciplinas relacionadas com saúde pública são ofertadas.

A necessidade de uma formação mais sólida em saúde pública é um tema explorado há bastante tempo e apontado como uma tendência da educação veterinária, visto o significativo aumento populacional mundial, o que torna essencial o desenvolvimento de 
uma medicina veterinária populacional (BÖGEL, 1992). Um ensino que desenvolva as competências humanísticas foi proposto pelo Conselho Federal de Medicina Veterinária e já vem sendo desenvolvido por algumas escolas de medicina veterinária. A atenção à saúde é um dos pontos requeridos, fazendo que o estudante tenha capacidade de compreender e perceber a saúde como um todo (LEMOS et al., 2015). O profissional deve possuir habilidade para trabalhar de forma interdisciplinar, para auxiliar as populações humanas a enfrentarem seus principais desafios (PFUETZENREITER; ZYLBERSZTAJN, 2004).

O acréscimo de maior carga horária voltada para saúde pública, no curso de medicina veterinária da UFPEL, poderia ser uma alternativa para qualificar a formação do médico veterinário. Porém, a abordagem dos temas relativos à saúde pública de forma transversal nas diversas disciplinas do curso, poderia elevar a qualidade da atuação do profissional que vai se especializar em outra área, além de criar elementos que sensibilizem mais adequadamente o estudante de veterinária para pensar no Sistema Único de Saúde e a atuação em saúde púbica. Da mesma forma, a inserção dos acadêmicos em atividades ligadas ao serviço de saúde, prático/teóricas, que evidenciam as questões que envolvem o tema no dia-a-dia da atuação profissional tem resultados favoráveis como demonstrado em outros cursos (ALBUQUERQUE et al., 2008). Ainda, a estratégia de imersão na realidade, através das práticas integrais, aplicada em ambientes multiprofissionais, traz a experimentação da realidade pelo discente, tornando-o mais crítico e sensível as questões que perpassam os diversos extratos da população, sensibilizando-o para as questões voltadas ao ambiente e à saúde pública, tão necessárias ao processo de formação contemporânea (TOMMASINO, 2008).

\section{CONCLUSÃO}

Os resultados evidenciaram que os acadêmicos de medicina veterinária da Universidade Federal de Pelotas estão cientes da relevância da profissão para a saúde pública, afirmando a sua importância, mas desconhecem alguns conceitos e como se dá sua atuação nessa área. A evolução esperada dos acadêmicos no decorrer do curso não foi evidenciada, permitindo concluir que a inserção curricular dos conteúdos da área é insuficiente. 


\section{PERCEPTION OF THE MEDICINE VETERINARY STUDENTS ABOUT THE TRAINING AND PRATICE IN PUBLIC HEALTH INSIDE THE UNIVERSIDADE FEDERAL DE PELOTAS-RS}

\section{ABSTRACT}

A $s$ the veterinary medicine's responsibility in contributing to the human population welfare increases, preparing the students to work in public health becomes a necessity. In this paper, we shall analyze the formation in public health through the students' perception of veterinary physicians' work in this field. The student's views were collected through an individual survey. The research shows the students are mostly women and the main reason for choosing the course is because they admire the veterinarian career. The students recognize the veterinary medicine's importance to the public health, but they do not know how that is presented in the undergraduate curriculum. The results did not show that learning was improved throughout the graduation, demonstrating the need to reevaluate the course's structure. It is vital that the students get access to that field during their formation years for the profession to occupy its rightful place in public health, through pedagogical strategies that include the student in the health service

Keywords: Teaching. Graduation. Public health. Health education.

\section{PERCEPCIÓN DE LOS ESTUDIANTES DE MEDICINA VETERINARIA SOBRE LA FORMACIÓN Y ACTUACIÓN EN SALUD PÚBLICA EN ÁMBITO DE LA UNIVERSIDADE FEDERAL DE PELOTAS-RS}

\section{RESUMEN}

a responsabilidad de la medicina veterinaria para contribuir a promover el bienestar de las poblaciones humanas es cada vez mayor, por lo que la preocupación por la formación académica en la salud pública es necesaria. Este estudio tuvo como objetivo analizar la educación en la salud pública, a través de la percepción de los estudiantes de medicina veterinaria sobre el papel del veterinario en el área de salud. Para identificar las nociones de estudiantes se aplicó cuestionarios individuales a los académicos. La investigación ha demostrado que el perfil de los estudiantes se compone sobre todo de las mujeres y la admiración por la carrera es la razón principal para elegir el curso. Los académicos reconocen la importancia de la profesión en la salud pública, pero no saben cómo es esta acción y que este tema se inserta en el plan de estudios de la carrera. No hubo evolución del aprendizaje a lo largo del curso, lo que demuestra la necesidad de reestructurar el currículo de la carrera. Es necesario que los egresados adquieran el 
conocimiento durante la etapa de preparación para la profesión con estrategias de enseñanza que incluyen al estudiante en el servicio de salud.

Palabras clave: Enseñanza. Graduación. Educación en Salud. Salud pública.

\section{REFERÊNCIAS}

ALBUQUeRQUe, V. S.; GOMES, A. P.; REZENDEI, C. H. A.; SAMPAIO, M. X.; DIAS, O. V.; LUGARINHO, R. M. A integração ensino-serviço no contexto dos processos de mudança na formação superior dos profissionais da saúde. Revista Brasileira de Educação Médica, v. 32, n. 3, p. 356-362, 2008.

BÖGEL, K. Veterinary public health perspectives: trend assessment and recommendations. Revue Scientific Technique, v. 11, n. 1, p. 219-239, 1992.

BRASIL. Conselho Nacional de Educação. Câmara de Educação Superior. Resolução no 1/03 de 20 de fevereiro de 2003. Institui diretrizes curriculares nacionais dos cursos de graduação em Medicina Veterinária. In: Diário Oficial da União, Brasília, n. 37, p. 15-16, 2003.

BRASIL. Ministério da Saúde. Portaria no 2.488 de 21 de outubro de 2011. Aprova a Política Nacional de Atenção Básica, estabelecendo a revisão de diretrizes e normas para a organização da Atenção Básica, para a Estratégia Saúde da Família (ESF) e o Programa de Agentes Comunitários de Saúde (PACS). In: Diário Oficial da União, Brasília, seção 1, n. 204, p. 48-55, 2011.

BÜRGER, K. P. O ensino de saúde pública veterinária nos cursos de graduação em medicina veterinária do Estado de São Paulo. Botucatu: UNESP, 2010. 129p. Tese (Doutorado em Medicina Veterinária), Faculdade de Ciências Agrárias e Veterinárias, Universidade Estadual Paulista, 2010.

CFMV - CONSELHO FEDERAL DE MEDICINA VETERINÁRIA. Médicas veterinárias e Zootecnistas estão cada vez mais atuantes na profissão, 2015. Disponível em: <http://portal.cfmv.gov.br/portal/noticia/4142>. Acesso em: 11 de julho de 2017.

EEFFTO - ESCOLA DE EDUCAÇÃO FÍSICA, FISIOTERAPIA E TERAPIA OCUPACIONAL/UFMG. Levantamento da UFMG indica transformações no perfil discente após adesão ao SISU, 2015. Disponível em:

<http://www.eeffto.ufmg.br/eeffto/noticias/95/levantamento_da_ufmg_indica_transforma caes_no_perfil_discente_apas_ad?lng=1>. Acesso em: 10 de julho de 2017.

LEMOS, R. S.; YAMANAKA, A. R.; CAMARGO, L. M.; et al. Desafios da educação. Revista CFMV, v. 21, n. 64, p. 23-31, 2015. 
MARCONI, M. A.; LAKATOS, E. M. Fundamentos de metodologia científica. 7. ed. São Paulo: Atlas, 2010.

MONDADORI, R. G.; HENRIQUE, B. S.; PIANTA, C.; et al. A trajetoria da mulher nos cursos de medicina veterinária no Brasil. Revista CFMV, v. 19, n. 58, p. 19-21, 2013.

PFUETZENREITER, M. R. O ensino da Medicina Veterinária Preventiva e Saúde Pública nos cursos de Medicina Veterinária - estudo de caso realizado na Universidade do Estado de Santa Catarina. Florianópolis: UFSC, 2003. 459p. Tese (Doutorado em Educação), Centro de Ciências da Educação, Programa de Pós-Graduação em Educação, Universidade Federal de Santa Catarina, 2003.

PFUETZENREITER, M. R.; ZYLBERSZTAJN, A. O ensino de saúde e os currículos dos cursos de medicina veterinária: um estudo de caso. Interface - Comunicação, Saúde, Educação, v. 8, n. 15, p. 349-360, 2004.

PFUETZENREITER, M. R.; ZYLBERSZTAJN, A. Percepções de estudantes, professores e médicos veterinários sobre o ensino da Medicina Veterinária Preventiva e Saúde Pública. Revista de Ciências Agroveterinárias, Lages, v. 7, n. 1, p. 75-84, 2008.

RAMOS, L. H. M. A Importância do Médico Veterinário na Vigilância Sanitária: Inserção e Participação na Gestão. In: SEMINÁRIO DE SAÚDE PÚBLICA VETERINÁRIA, 2008, São Paulo. RESUMOS. São Paulo: CFMV/CRMVs, 2008.

SABROZA, P. C. Saúde Pública: procurando os limites da crise. In: CONFERÊNCIA PANAMERICANA DE EDUCAÇÃO EM SAÚDE PÚBLICA, 1994, Rio de Janeiro. RESUMOS. Rio de janeiro: Escola Nacional de Saúde Pública, Fundação Oswaldo Cruz, 1994.

SOCIAL SCIENCE STATISTICS. Disponível em:

<http://www.socscistatistics.com/tests/chisquare2/Default2.aspx> . Acesso em: 10 de março de 2017.

TOMMASINO, H. Generalizacion de las prácticas integrales - los aportes de la extensión para su implementación, 2008. Disponível em:

<http://psico.edu.uy//sites/default/files/tommasino_practicas_integrales_0.pdf> . Acesso em: 11 de julho de 2017.

Autor para correspondência:

Alessandra Jacomelli Teles.

Universidade Federal de Pelotas, Faculdade de Veterinária, Campus Universitário, Capão do Leão (RS), CEP

96010-900, Brasil.

ale.teles@gmail.com 